\title{
Human Enterocytes as an In Vitro Model for the Evaluation of Intestinal Drug Metabolism: Characterization of Drug-Metabolizing Enzyme Activities of Cryopreserved Human Enterocytes from Twenty-Four Donors
}

\author{
Received November 22, 2016; accepted April 5, 2017
}

\begin{abstract}
We report in this work successful isolation and cryopreservation of enterocytes from human small intestine. The enterocytes were isolated by enzyme digestion of the intestinal lumen, followed by partial purification via differential centrifugation. The enterocytes were cryopreserved directly after isolation without culturing to maximize retention of in vivo drug-metabolizing enzyme activities. Post-thaw viability of the cryopreserved enterocytes was consistently over $\mathbf{8 0} \%$ based on trypan blue exclusion. Cryopreserved enterocytes pooled from eight donors (four male and four female) were evaluated for their metabolism of 14 pathway-selective substrates: CYP1A2 (phenacetin hydroxylation), CYP2A6 (coumarin 7-hydroxylation), CYP2B6 (bupropion hydroxylation), CYP2C8 (paclitaxel $6 \alpha$-hydroxylation), CYP2C9 (diclofenac 4-hydroxylation), CYP2C19 (S-mephenytoin 4-hydroxylation), CYP2D6 (dextromethorphan
\end{abstract}

Introduction

Intestinal metabolism has been reported to be responsible for the low bioavailability of approximately $50 \%$ orally-administered drugs (Watkins, 1992; Wacher et al., 2001; Kaminsky and Zhang, 2003; Thummel, 2007). In vitro evaluation of intestinal drug metabolism therefore represents an experimental approach that can be applied to guide the development of drugs with acceptable oral bioavailability. The commonly used in vitro model of intestinal mucosal epithelium, the colon adenocarcinoma $\mathrm{Caco} 2$ cell line, although useful in defining permeability and P-gp efflux, is generally not considered appropriate for the evaluation of enteric drug metabolism due to the low basal level of drug-metabolizing enzyme (DME) activity (Prueksaritanont et al., 1996; Nakamura et al., 2002). To overcome this deficiency, Caco 2 cells have been transfected with human cytochrome P450 (P450) isoforms, especially CYP3A4, for the evaluation of drug bioavailability in the presence of P450 metabolism (Crespi et al., 1996; Küblbeck et al., 2016). However, this approach is far from representative of intestinal metabolism, which is known to involve multiple pathways, including various P450 isoforms and non-P450 DME (Paine et al., 2006; Nakamura et al., 2016).

Current in vitro experimental models of human intestinal metabolism include human intestinal microsomes (Kolars et al., 1992; Galetin et al.,

https://doi.org/10.1124/dmd.116.074377. hydroxylation), CYP2E1 (chlorzoxazone 6-hydroxylation), CYP3A4 (midazolam 1'-hydroxylation and testosterone $6 \beta$-hydroxylation), CYP2J2 (astemizole 0-demethylation), UDP-glucuronosyltransferase (UGT; 7-hydroxycoumarin glucuronidation), sulfotransferase (SULT; 7-hydroxycoumarin sulfation), and carboxylesterase 2 (CES2; irinotecan hydrolysis) activities. Quantifiable activities were observed for CYP2C8, CYP2C9, CYP2C19, CYP2E1, CYP3A4, CYPJ2, CES2, UGT, and SULT, but not for CYP1A2, CYP2A6, CYP2B6, and CYP2D6. Enterocytes from all 24 donors were then individually evaluated for the quantifiable drug metabolism pathways. All demonstrated quantifiable activities with the expected individual variations. Our results suggest that cryopreserved human enterocytes represent a physiologically relevant and convenient in vitro experimental system for the evaluation of intestinal metabolism, akin to cryopreserved human hepatocytes for hepatic metabolism.
2008), precision cut intestinal slices (van de Kerkhof et al., 2008), and freshly isolated enterocytes (Bader et al., 2000; Hansen et al., 2000; Zhang et al., 2003; Bonnefille et al., 2011). Of these systems, primary enterocytes may represent the most physiologically relevant model, akin to primary hepatocytes for hepatic metabolism. Intact enterocytes possess key cellular properties that are key to the assessment of in vivo events, including an intact plasma membrane to allow modeling of membrane permeability, uptake and efflux drug transporters, as well as complete and uninterrupted DME pathways and cofactors for both phase I oxidation and phase II conjugation.

In our laboratory, we embarked upon the isolation and cryopreservation of enterocytes with the goal of developing a physiologically relevant experimental model for the evaluation of intestinal uptake, metabolism, and efflux. We report in this work our success in the isolation and cryopreservation of human enterocytes to retain viability and drug metabolism enzyme activities.

\section{Materials and Methods}

Chemicals. Astemizole, irinotecan hydrochloride, dextrorphan tartrate, diclofenac sodium salt, 7-ethyl-10-hydroxycamptothecin (SN38), 4-hydroxydiclofenac, $S$-mephenytoin, 4-hydroxymephenytoin, paclitaxel, and testosterone were purchased from Cayman Chemical (Ann Arbor, MI). The 7-hydroxycoumarin was purchased from Chem Service (West Chester, PA). Bupropion hydrochloride was obtained from AK Scientific (Union City, CA). The 7-hydroxycoumarin sulfate

ABBREVIATIONS: CES, carboxylesterase; DME, drug-metabolizing enzyme; HQM, hepatocyte/enterocyte incubation medium; P450, cytochrome P450; SULT, sulfotransferase; UGT, UDP-glucuronosyltransferase. 
potassium salt was obtained from Santa Cruz Biotechnology (Dallas, TX). Chlorzoxazone, coumarin, dextromethorphan hydrobromide, $6 \beta$-hydroxytestosterone, 7-hydroxycoumarin $\beta$-D-glucuronide sodium salt, (2S, 3S)-hydroxy bupropion hydrochloride, 7-ethoxycoumarin, and phenacetin were purchased from SigmaAldrich (St. Louis, MO). The 6-hydroxychlorzoxazone, $6 \alpha$-hydroxypaclitaxel, midazolam, 1'-hydroxymidazolam, O-desmethyl astemizole, and 4-hydroxy-Smephenytoin were obtained from Toronto Research Chemicals (Toronto, Canada).

Human Intestine. Human intestines from multiple donors were obtained from the International Institute for the Advancement of Medicine (IIAM, Exton, PA).

Enterocyte Isolation and Cryopreservation. Isolation of enterocytes from human intestines was performed via enzymatic digestion of the intestinal lumen based on procedures previously reported for porcine intestines (Bader et al., 2000; Hansen et al., 2000). The following lengths (postpyloric sphincter) were used to aid the identification of the various regions of the small intestine for enterocyte isolation: duodenum, $26 \mathrm{~cm}$ (9.84 inches); jejunum, $2.5 \mathrm{~m}$ ( 8.2 feet); and ileum, $3.5 \mathrm{~m}$. The intestines were recovered with a warm ischemic time of less than 15 minutes and shipped to our laboratory on wet ice in University of Wisconsin Preservation Solution, with a cold ischemic time of less than 24 hours.

Upon receipt of the small intestines, adipose tissue associated with intestines was removed by dissection. The intestinal lumen was washed rapidly with cold calcium and magnesium-free Hank's balanced salt solution to remove intestinal contents, followed by digestion with an isotonic buffer containing $0.25 \mathrm{mg} / \mathrm{mL}$ type I collagenase (Sigma-Aldrich). The cells released from the intestinal lumen were sieved to remove relatively large cell clusters, followed by partial purification by differential centrifugation $(100 g, 20$ minutes). The enterocytes were quantified and cryopreserved immediately after isolation using a programmable liquid nitrogen cell freezer and stored in the vapor phase of liquid nitrogen maintained at $<-150^{\circ} \mathrm{C}$.

Recovery of Cryopreserved Enterocytes. Cryopreserved enterocytes (In Vitro ADMET Laboratories, Columbia, MD) were thawed in a $37^{\circ} \mathrm{C}$ water bath for approximately 2 minutes and transferred by pouring into a $50 \mathrm{ml}$ conical of cryopreserved enterocyte recovery medium (In Vitro ADMET Laboratories) that was prewarmed in a $37^{\circ} \mathrm{C}$ water bath. The thawed enterocytes were recovered by centrifugation at $100 \mathrm{~g}$ for 10 minutes at room temperature. After centrifugation, the supernatant was removed by decanting. A volume of $250 \mu 14^{\circ} \mathrm{C}$ hepatocyte/enterocyte incubation medium (HQM; In Vitro ADMET Laboratories) was added to the intact pellet of enterocytes at the bottom of the conical tube, followed by gentle agitation to reconstitute an enterocyte suspension. Viability and yield were quantified in a hemacytometer based on trypan blue dye exclusion (Sigma-Aldrich).

Measurement of Enterocyte Diameter. Photomicrographs of the enterocytes were taken using a phase-contrast photomicroscope. The photomicrographs were printed, and the diameters were measured and corrected for the magnification factor. Results $(\mu \mathrm{m})$ are expressed as mean and standard deviation values of 50 randomly chosen cells from each enterocyte lot.
Incubation of Enterocytes with DME Substrates. DME substrate incubations were performed in a cell culture incubator maintained at $37^{\circ} \mathrm{C}$ with a humidified atmosphere of $5 \% \mathrm{CO}_{2}$. Enterocyte cell density was adjusted to $3 \times 10^{6}$ cells $/ \mathrm{ml}$ in HQM. Aliquots of $50 \mu \mathrm{l}$ cell suspension were added to individual wells $(150,000$ viable enterocytes/well) of a 96-well plate for the evaluation of drug metabolism activities. After cell addition, the 96-well plate was prewarmed in the incubator for 15 minutes, followed by the addition of $50 \mu \mathrm{l}$ prewarmed $\left(37^{\circ} \mathrm{C}\right) \mathrm{HQM}$ containing DME substrates at $2 \times$ final concentration and incubated for 2 hours. The final incubation mixture in each well therefore had a volume of $100 \mu \mathrm{l}$, with a cell density of $1.5 \times 10^{6}$ cells $/ \mathrm{ml}$. Substrates for the multiple drug metabolism pathways evaluated are shown in Table 1. Metabolism was terminated in each well by the addition of $100 \mu \mathrm{l}$ acetonitrile. The final incubation samples were stored at $-80^{\circ} \mathrm{C}$ for the subsequent LC/MS-MS analysis.

LC/MS-MS Quantification of Metabolite Formation. Upon thawing, an aliquot of $100 \mu \mathrm{l}$ acetonitrile containing an internal standard of $250 \mathrm{nM}$ tolbutamide was added to each sample. All samples were centrifuged at $13,000 \mathrm{rpm}$ for 5 minutes. An aliquot of $100 \mu \mathrm{L}$ supernatant from each was transferred to a 96-well plate and was diluted with $200 \mu \mathrm{L}$ deionized water for LC/MS-MS analysis using an API 4000 QTRAP mass spectrometer with an electrospray ionization source (AB SCIEX, Framingham, MA) connected to Agilent 1200 series high-pressure liquid chromatography (Agilent Technologies, Santa Clara, CA). An Agilent Zorbax Eclipse Plus C18 column $(4.6 \times 75 \mathrm{~mm}$ i.d., $3.5 \mu \mathrm{m}$; Agilent Technologies) at a flow rate of $1 \mathrm{~mL} / \mathrm{min}$ was used for the chromatography separation. The mobile phase consisted of $0.1 \%$ formic acid in acetonitrile (A) and $0.1 \%$ formic acid in water (B). The gradient for the positive ion mode operation was programmed as follows: 0 to 2.5 minutes, increase $B$ from 5 to $95 \% ; 2.5$ to 3.5 minutes, $95 \% \mathrm{~B} ; 3.5$ to 3.6 minutes, decrease $\mathrm{B}$ to $5 \%$; run time 5 minutes. The gradient program for the negative ion mode was as follows: 0 to 3 minutes, increase B from 5 to $95 \% ; 3$ to 4 minutes, $95 \%$ B; 4 to 4.2 minutes, decrease $\mathrm{B}$ to $5 \%$; run time 6 minutes. Data acquisition and data procession were performed with the software Analyst 1.6.2 (AB SCIEX). Standard assays of the metabolites were performed in LC/MS-MS mass transition monitoring mode, monitoring the mass transitions (parent to daughter ion). The metabolism substrates used, identities of the metabolites quantified, and LC/MS-MS parameters are shown in Table 1.

\section{Results}

Donor Demographics. Enterocytes were isolated and cryopreserved from 25 donors. Age, gender, and ethnicity of the donors are shown in Table 2.

Morphology, Size, Viability, and Yield of Cryopreserved Human Enterocytes. The morphology of cryopreserved human enterocytes immediately after recovery is shown in Fig. 1. The enterocytes consisted

TABLE

Metabolic pathways, substrates, metabolites, and LC/MS-MS parameters for the quantification of the DME activities of cryopreserved human enterocytes

Tolbutamide was used as an internal standard with the mass transition monitoring at $\mathrm{m} / \mathrm{z} 271.2$ to $91.3 \mathrm{and} \mathrm{m} / \mathrm{z} 269.1$ to 105.9 for positive mode and negative mode, respectively.

\begin{tabular}{|c|c|c|c|c|}
\hline Metabolic Pathway & Pathway-Selective Substrate $(\mu \mathrm{M})$ & Marker Metabolite & Ion Mode Application & Mass Transition Monitoring \\
\hline CYP1A2 & Phenacetin (100) & Acetaminophen & Positive & $\mathrm{m} / \mathrm{z} 152.1$ to 109.9 \\
\hline CYP2A6 & Coumarin $(50)$ & 7-Hydroxycoumarin & Negative & $\mathrm{m} / \mathrm{z} 161.0$ to 132.9 \\
\hline CYP2B6 & Bupropion (500) & Hydroxybupropion & Positive & $\mathrm{m} / \mathrm{z} 250.1$ to 130.1 \\
\hline CYP2C8 & Paclitaxel (20) & $6 \alpha$-Hydroxypaclitaxel & Positive & $\mathrm{m} / \mathrm{z} 870.4$ to 525.2 \\
\hline CYP2C9 & Diclofenac (25) & 4-Hydroxydiclofenac & Negative & $\mathrm{m} / \mathrm{z} 309.8$ to 265.9 \\
\hline CYP2C19 & $S$-Mephenytoin (250) & 4-Hydroxy-S-Mephenytoin & Positive & $\mathrm{m} / \mathrm{z} 235.2$ to 150.0 \\
\hline CYP2D6 & Dextromethorphan (15) & Dextrophan & Positive & $\mathrm{m} / \mathrm{z} 258.1$ to 157.1 \\
\hline CYP2E1 & Chlorzoxazone (250) & 6-Hydroxychlorzoxazone & Negative & $\mathrm{m} / \mathrm{z} 183.9$ to 119.8 \\
\hline CYP3A4-1 & Midazolam (20) & 1'-Hydroxymidazolam & Positive & $\mathrm{m} / \mathrm{z} 342.1$ to 203.1 \\
\hline CYP3A4-2 & Testosterone (200) & $6 \beta$-Hydroxytestosterone & Positive & $\mathrm{m} / \mathrm{z} 305.2$ to 269.1 \\
\hline CYP2J2 & Astemizole (50) & O-Demethylastemizole & Positive & $\mathrm{m} / \mathrm{z} 445.0$ to 204.2 \\
\hline CES2 & Irinotecan $(50)$ & SN38 & Positive & $\mathrm{m} / \mathrm{z} 393.0$ to 349.3 \\
\hline UGT & 7-Hydroxycoumarin (100) & 7-Hydroxycoumarin Glucuronide & Negative & $\mathrm{m} / \mathrm{z} 336.9$ to 160.9 \\
\hline SULT & 7-Hydroxycoumarin (100) & 7-Hydroxycoumarin Sulfate & Negative & $\mathrm{m} / \mathrm{z} 240.9$ to 161.0 \\
\hline
\end{tabular}


TABLE 2

Demographics, viability, yield, and cellular diameter of human enterocytes from 25 individual donors

\begin{tabular}{|c|c|c|c|c|c|c|c|c|}
\hline Lot Number & Gender & Ethnicity & Age (years) & BMI & Viability (\%) & Yield (million) & Cellular Diameter $(\mu \mathrm{m})$ & Region \\
\hline HE3005 & Male & Caucasian & 23 & 25 & 88 & 2.8 & 15.6 & Duodenum \\
\hline HE3006 & Female & Caucasian & 44 & 32.7 & 78 & 0.9 & 15.5 & Duodenum \\
\hline HE3008 & Male & Hispanic & 43 & 30.3 & 80 & 1.5 & 14.3 & Duodenum \\
\hline HE3009 & Male & Caucasian & 18 & 29.5 & 84 & 2.7 & 15 & Duodenum \\
\hline HE3010 & Female & Caucasian & 44 & 48.3 & 89 & 2.8 & 15 & Duodenum \\
\hline HE3011 & Male & Caucasian & 47 & 31.1 & 85 & 1.5 & 14.2 & Duodenum \\
\hline HE3012 & Female & Caucasian & 50 & 24.2 & 79 & 1.2 & 15.4 & Duodenum \\
\hline HE3013 & Female & African American & 57 & 33.0 & 75 & 1 & 14.8 & Duodenum \\
\hline HE3014 & Male & African American & 49 & 24.1 & 89 & 0.7 & 14.5 & Duodenum \\
\hline HE3015 & Male & Caucasian & 24 & 26.1 & 86 & 2.4 & 14.8 & Duodenum \\
\hline HE3016 & Male & African American & 32 & 23.4 & 89 & 1.4 & 15.4 & Duodenum \\
\hline HE3019 & Male & Caucasian & 61 & 30 & 89 & 0.7 & 15.4 & Duodenum \\
\hline HE3020 & Male & Caucasian & 25 & 24.8 & 89 & 1.2 & 14.6 & Duodenum \\
\hline HE3021 & Male & African American & 60 & 20.7 & 88 & 0.7 & 12.9 & Duodenum \\
\hline HE3027 & Female & Caucasian & 53 & 36.5 & 85 & 1.6 & 14.4 & Duodenum \\
\hline HE3028 & Male & African American & 34 & 30.6 & 86 & 1.3 & 15.3 & Duodenum \\
\hline HE3029 & Male & Caucasian & 41 & 34.6 & 85 & 1.7 & 13.4 & Duodenum \\
\hline HE3030 & Male & African American & 38 & 21.1 & 78 & 0.7 & 14.5 & Duodenum \\
\hline HE3031 & Female & Caucasian & 49 & 25.5 & 86 & 1 & 12.5 & Duodenum \\
\hline HE3032 & Female & Caucasian & 48 & 27.1 & 78 & 1.7 & 15.3 & Duodenum \\
\hline HE3033 & Male & Hispanic & 32 & 21 & 85 & 3.7 & 15.2 & Duodenum \\
\hline HE3034 & Female & Caucasian & 53 & 18.8 & 95 & 3.5 & 17.2 & Duodenum \\
\hline HE3040 & Male & African American & 38 & 21.1 & 86 & 2.5 & 15.6 & Duodenum \\
\hline HE3042 & Male & Caucasian & 60 & 22.8 & 85 & 2 & 16.2 & Duodenum \\
\hline HE3043 & Male & Caucasian & 43 & 26.3 & 81 & 2 & 15 & Duodenum \\
\hline
\end{tabular}

BMI, body mass index.

of either single cells or small cell clusters, with a rounded morphology typical of mammalian cells in suspension. The average post-thaw viability of human enterocytes for the 25 donors was $84.7 \pm 4.7 \%$ with a range of $78 \%$ to $95 \%$, with an average diameter of $14.9 \pm 1.0 \mu \mathrm{m}$ (Table 2).

\section{DME Activities}

Metabolism of 14 Substrates Using Pooled Human Enterocytes. Human enterocytes from four male and four female donors were combined (pooled) and incubated with 14 pathway-selective substrates for the evaluation of their drug metabolism potential. Results are shown in Table 3.

Individual Variations in Drug-Metabolizing Enzyme Activities. Enterocytes from 24 donors were evaluated for CYP2C9 (diclofenac 4-hydroxylation), CYP2C19 (S-mephenytoin 4-hydroxylation), CYP3A4 (midazolam 1 '-hydroxylation and testosterone $6 \beta$-hydroxylation), CYP2J2 (astemizole O-demethylation), carboxylesterase (CES; irinotecan hydrolysis), UDP-glucuronosyltransferase (UGT; 7-hydroxycoumarin glucuronidation), and sulfotransferase (SULT; 7-hydroxycoumarin sulfation) activities. Results are shown in Table 4.

\section{Discussion}

Intestinal uptake, efflux, and metabolism are the three key determinants of bioavailability of orally administered drugs. Enterocytes isolated from the small intestines represent an ideal model to evaluate these determinants. Successful isolation of primary enterocytes to retain DME activities has been previously reported using a variety of procedures, including the following: from mice via EDTA perfusion (Zhang et al., 2003), from pigs via collagenase digestion (Bader et al., 2000; Hansen et al., 2000), and from humans via mechanical separation (Chougule et al., 2012). Our work represents the first report of successful cryopreservation of primary enterocyte isolates from humans to retain viability and DME activities. Using enterocytes pooled from four male and four female donors, quantifiable metabolite formation was observed for CYP2C8 (paclitaxel $6 \alpha$-hydroxylation), CYP2C9 (diclofenac 4-hydroxylation), CYP2C19 ( $S$-mephenytoin 4-hydroxylation), CYP2E1 (chlorzoxazone 6-hydroxylation), CYP3A4 (midazolam 1'hydroxylation and testosterone $6 \beta$-hydroxylation), CYP2J2 (astemizole O-demethylation), CES (irinotecan hydrolysis), UGT, and SULT. Undetectable activities were observed for the following DME: CYP1A2 (phenacetin hydroxylation), CYP2A6 (coumarin 7-hydroxylation), CYP2B6 (bupropion hydroxylation), and CYP2D6 (dextromethorphan hydroxylation). Ranking of activities in descending order is as follows: CYP3A4 (testosterone) $>$ SULT $>$ UGT $>$ CYP3A4 (midazolam) $>$ CYP2C9 $>$ CYP2J2 $>$ CYP2C19 = CYP2E1 $\geq$ CES2 $\geq$ CYP2C8. Our ranking of the quantifiable DME in enterocytes is consistent with that reported by others for P450 (Paine et al., 2006; Xie et al., 2016), UGT (Radominska-Pandya et al., 1998; Bock, 2016), and SULT (Chen et al., 2003; Teubner et al., 2007), with CYP3A4 and UGT believed to be the most important DME responsible for metabolism-dependent enteric drug bioavailability. One interesting observation is lack of significant CYP2D6 activity in the intestines, as this P450 isoform is known to be specifically responsible for hepatic metabolism of a large number of commonly used human drugs, especially for antipsychotic drugs (Vandel et al., 1999). The lack of CYP2D6 activities is consistent with the findings with oxycodone metabolism by intestinal mucosal microsomes in which the CYP3A4-mediated $N$-demethylation, but not the CYP2D6-mediated $O$-demethylation, was observed (Lalovic et al., 2004).

Based on the results with the pooled enterocytes, enterocytes from 24 donors were evaluated to estimate the extent of interindividual variations in enteric drug metabolism. The 24 donors represent the enterocytes that we successfully isolated and cryopreserved at the time of the preparation of this manuscript. The results confirmed that enterocytes possess CYP2C9, CYP2C19, CYP3A4, CYP2J2, CES2, UGT, and SULT activities, with substantial interindividual differences. The range of activities, expressed as $\mathrm{pmol} / \mathrm{min} /$ million enterocytes, for the various DME are as follows: CYP2C9, 0.03 (HE3011)-7.93 (HH3034); CYP2C19, 0.01 (HE3011)-1.13 


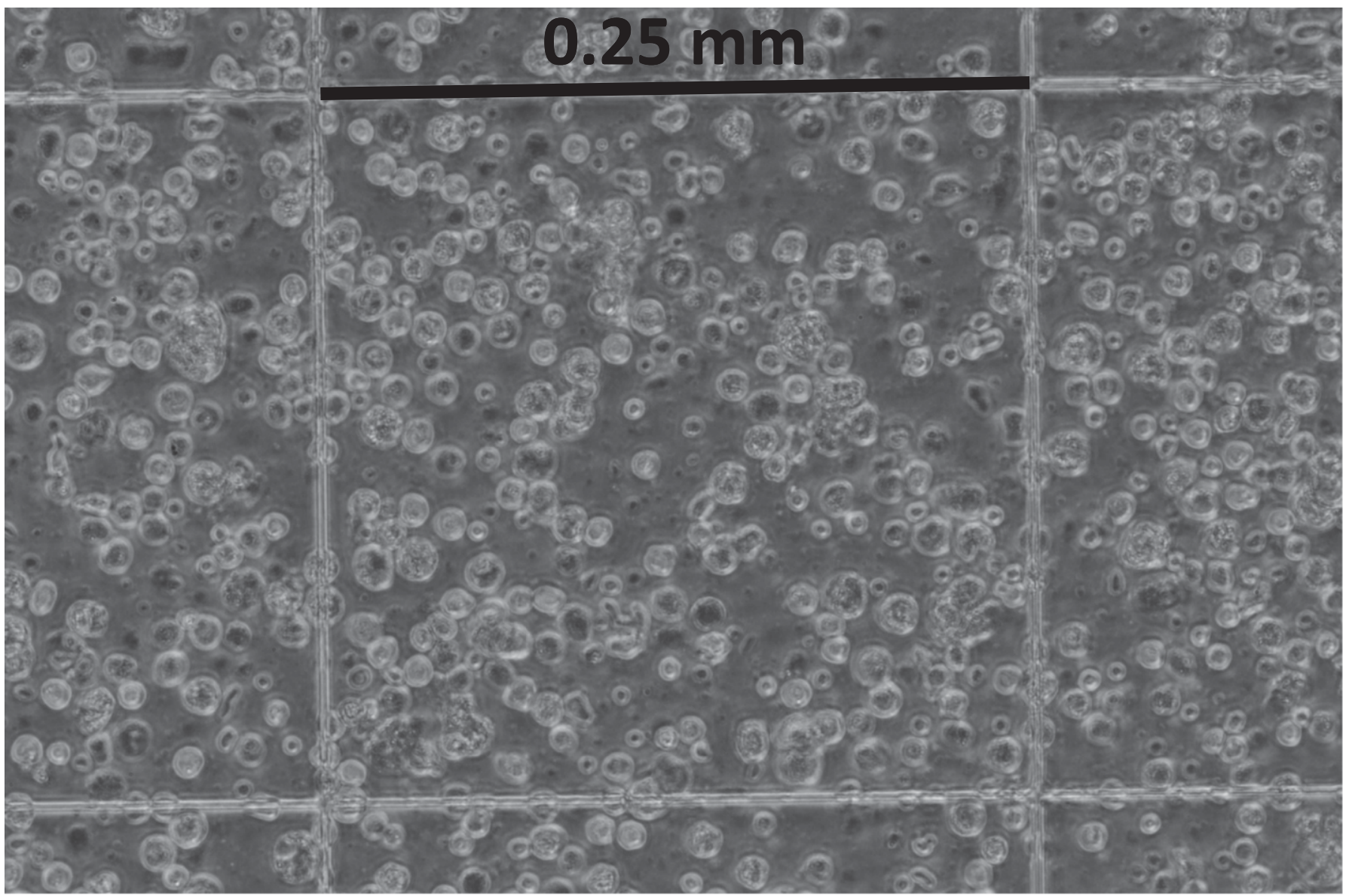

Fig. 1. Phase-contrast microscopy of cryopreserved and thawed human enterocytes. Enterocytes from HE3005, a 23-year-old male Caucasian, were shown. The cells exhibited rounded cell morphology with uniform cell size. The enterocytes were mainly single cells with some cell aggregates.

(HE3033); CYP3A4 (midazolam 1'-hydroxylation), 0.09 (HE3011)4.35 (HH3034); CYP3A4 (testosterone 6 $\beta$-hydroxylation), 2.60 (HE3011)-45.2 (HE3034); CYP2J2, 0.19 (HE3021)-1.98 (HE3034); CES2, 0.05 (HE3030)-0.60 (HE3009); UGT, 1.01 (HE3011)-122.6 (HE3034); and SULT, 0.79 (HE3031)-22.0 (HE3040). An interesting observation is that one of the enterocyte lots, HE3034 (from a 50-year-old female Caucasian), was found to have UGT activity of $122.6 \mathrm{pmol} / \mathrm{min} / \mathrm{million}$ enterocytes, approximately 12 -fold of the average of the values for enterocytes from the other 23 donors (ranging from 1.01 to $29.6 \mathrm{pmol} / \mathrm{min} / \mathrm{million}$ enterocytes) of $9.7 \mathrm{pmol} / \mathrm{min} / \mathrm{million}$ enterocytes. This same donor also had highest CYP3A4 and CYP2J2 activities. In contrast, HE 3011 (from a 53-year-old female Caucasian) had the lowest activities for CYP2C9, CYP2C19, CYP3A4, and UGT activities. Individual differences in intestinal metabolism are a potential determinant in individual differences in bioavailability for orally administered drugs (Jamei et al., 2009). Our results suggest that enterocytes from individuals with different DME activities may be used to evaluate this important drug property. However, it is to be added that cryopreservation may lead to attenuation of DME activities. This possibility is being investigated in our laboratory. For now, our data definitively demonstrate that the cryopreserved enterocytes were active in the DME activities evaluated. Whether the cryopreserved enterocyte reflects in vivo activities is yet to be determined.
TABLE 3

DME activities of cryopreserved human enterocytes

Enterocytes from 4 male (HE3005, HE3007, HE3008, HE3010) and 4 females (HE3006, HE3009, HE3011, HE3013) were pooled and used in the study. The DME pathways evaluated were as follows: CYP1A2 (phenacetin hydroxylation), CYP2A6 (coumarin 7-hydroxylation), CYP2B6 (bupropion hydroxylation), CYP2C8 (paclitaxel 6 $\alpha$-hydroxylation), CYP2C9 (diclofenac 4-hydroxylation), CYP2C19 (S-mephenytoin 4-hydroxylation), CYP2D6 (dextromethorphan hydroxylation), CYP2E1 (chlorzoxazone 6-hydroxylation), CYP3A4 [midazolam 1'-hydroxylation (CYP3A4M) and testosterone 6 $\beta$-hydroxylation (CYP3A4T)], CYP2J2 (astemizole O-demethylation), UGT (7-hydroxycoumarin glucuronidation), SULT (7-hydroxycoumarin sulfation), and CES2 (irinotecan hydrolysis) activities. Results are mean and S.D. of triplicate determinations. Activities for CYP1A2, CYP2A6, CYP2B6, and CYP2D6 were below quantifiable levels (BQL).

\begin{tabular}{lcc}
\hline & \multicolumn{2}{c}{ Specific Activity (pmol/min/million Enterocytes) } \\
\cline { 2 - 3 } DME Pathway & Mean & S.D. \\
\hline CYP1A2 & BQL & NA \\
CYP2A6 & BQL & NA \\
CYP2B6 & BQL & NA \\
CYP2C8 & 0.2 & 0 \\
CYP2C9 & 2.0 & 0.2 \\
CYP2C19 & 0.4 & 0.1 \\
CYP2D6 & BQL & NA \\
CYP2E1 & 0.4 & 0.1 \\
CYP3A4M & 2.8 & 0.5 \\
CYP3A4T & 19.2 & 5.8 \\
CYP2J2 & 1.6 & 0.1 \\
CES2 & 0.3 & 0 \\
UGT & 4.5 & 0.3 \\
SULT & 11.4 & 0.7 \\
\hline
\end{tabular}

NA, not applicable. 
TABLE 4

DME activities of cryopreserved human enterocytes from 24 donors

The mathematical average (mean) and S.D. of the 24 donors are calculated from the results of each individual donor. The DME pathways evaluated were as follows: CYP2C9 (diclofenac 4-hydroxylation), CYP2C19 (S-mephenytoin 4-hydroxylation), CYP3A4 [midazolam 1'-hydroxylation (CYP3A4M) and testosterone 6 $\beta$-hydroxylation (CYP3A4T)], CYP2J2 (astemizole O-demethylation), CES (irinotecan hydrolysis), UGT (7-hydroxycoumarin glucuronidation), and SULT (7-hydroxycoumarin sulfation) activities. Mean and S.D. values of the activities of the 24 donors are shown at the bottom of the table.

\begin{tabular}{|c|c|c|c|c|c|c|c|c|}
\hline \multirow{2}{*}{ Lot Number } & \multicolumn{8}{|c|}{ Activity (pmol/min/million Enterocytes) } \\
\hline & CYP2C9 & CYP2C19 & CYP $3 A 4 M$ & CYP3A4T & CYP2J2 & CES2 & $U G T$ & SULT \\
\hline HE3005 & 1.68 & 0.56 & 2.67 & ND & 1.2 & 0.23 & 8.38 & 8.72 \\
\hline HE3006 & 0.59 & 0.29 & 0.13 & 6.23 & 0.73 & 0.33 & 2.3 & 2.04 \\
\hline HE3007 & 0.91 & 0.39 & 0.99 & 22.7 & 0.57 & 0.46 & 3.08 & 4.04 \\
\hline HE3008 & 0.46 & 0.68 & 0.87 & NA & 0.33 & 0.55 & 1.8 & 1.79 \\
\hline HE3009 & 1.18 & 0.35 & 0.72 & 32.1 & 0.25 & 0.6 & 4.32 & 7.78 \\
\hline HE3010 & 1.21 & 0.62 & 0.46 & 17.5 & 0.99 & 0.41 & 2.56 & 3.32 \\
\hline HE3011 & 0.03 & 0.01 & 0.09 & 2.6 & 0.33 & 0.29 & 1.01 & 1.7 \\
\hline HE3014 & 0.44 & 0.11 & 0.4 & 8.2 & 1.18 & 0.17 & 3.55 & 2.66 \\
\hline HE3015 & 2.5 & 0.49 & 2.55 & ND & 0.95 & 0.51 & 7.33 & 5.23 \\
\hline HE3016 & 2.05 & 1.08 & 0.99 & 26.7 & 0.93 & 0.34 & 5.71 & 4.13 \\
\hline HE3019 & 0.24 & 0.11 & 0.3 & 5.27 & 0.26 & 0.3 & 1.47 & 1.89 \\
\hline HE3020 & 0.31 & 0.14 & 0.49 & 12.9 & 0.58 & 0.59 & 5.83 & 1.84 \\
\hline HE3021 & 0.2 & 0.06 & 0.17 & 2.97 & 0.19 & 0.08 & 1.49 & 1.64 \\
\hline HE3027 & 2.02 & 0.31 & 0.68 & 25.9 & 0.76 & 0.19 & 3.68 & 2.69 \\
\hline HE3028 & 0.68 & 0.21 & 0.82 & 26 & 0.71 & 0.3 & 3.84 & 9.02 \\
\hline HE3029 & 0.86 & 0.12 & 0.59 & 12.1 & 0.76 & 0.08 & 6.55 & 3.65 \\
\hline HE3030 & 0.26 & 0.28 & 0.29 & 14.9 & 0.32 & 0.05 & 15 & 8.3 \\
\hline HE3031 & 0.34 & 0.09 & 0.16 & 2.95 & 0.49 & 0.18 & 1.6 & 0.79 \\
\hline HE3032 & 1.84 & 0.81 & 1.3 & 43.4 & 0.82 & 0.24 & 5.9 & 4.5 \\
\hline HE3033 & 2.11 & 1.13 & 1.03 & 28.4 & 0.7 & 0.11 & 29.6 & 14.2 \\
\hline HE3034 & 7.93 & 0.71 & 4.35 & 45.2 & 1.98 & 0.12 & 122.6 & 8.24 \\
\hline HE3040 & 0.96 & 0.78 & 1.00 & 32.30 & 0.30 & 0.18 & 23.80 & 22.00 \\
\hline HE3042 & 0.23 & 0.03 & 1.66 & 31.20 & 1.06 & 0.06 & 74.20 & 8.98 \\
\hline HE3043 & 1.39 & 0.21 & 0.62 & 18.80 & 0.55 & 0.23 & 9.13 & 9.00 \\
\hline Mean & 1.27 & 0.40 & 0.97 & 19.92 & 0.71 & 0.28 & 14.36 & 5.76 \\
\hline S.D. & 1.59 & 0.33 & 0.99 & 12.99 & 0.41 & 0.17 & 27.74 & 4.88 \\
\hline
\end{tabular}

NA, not applicable; ND, not determined.

Besides the metabolic activities shown in this work, we have quantified gene expression of the enterocytes from the multiple donors and demonstrated that the cells consistently express enterocyte-specific markers, P450 isoforms, uptake, and efflux transporters (manuscript in preparation).

Cryopreserved enterocytes may represent the gold standard for enteric metabolism studies, as do human hepatocytes for hepatic metabolism (Fabre et al., 1990; Gómez-Lechón et al., 2004; Li, 2010), for similar reasons. An intact plasma membrane with active transporters allows the modeling of drug permeability. Complete, uninterrupted DME systems allow simultaneous evaluation of multiple metabolic pathways. CYP450 and phase II DME and their cofactors exist at physiologic concentrations in enterocytes, which minimizes experimental artifacts such as ubiquitous CYP450 protein binding observed in human liver microsomes. Finally, cytosolic proteins in enterocytes allow modeling of intracellular protein binding. Cryopreserved enterocytes may be used routinely in drug development to allow the optimization of drug candidates with the most appropriate enteric metabolic properties. Two major potential applications of cryopreserved human enterocytes are as follows: 1) investigation of the role of enteric metabolism on bioavailability of orally administered drugs; and 2) enteric drugdrug and food-drug interactions that occur specifically in the intestine but not in the liver, as exemplified by the findings with grapefruit juice on intestinal drug metabolism (Holmberg et al., 2014), uptake (Shirasaka et al., 2013), and efflux (Wang et al., 2001). A major focus of our laboratory currently is the development of experimental approaches using cryopreserved human enterocytes to evaluate key enteric drug properties, including metabolic clearance, metabolite profiling, transportermediated uptake and efflux, P450 inhibition and induction, and enterotoxicity.

In Vitro ADMET Laboratories, Columbia, Maryland

Ming-ChIH DAVID Ho NichOLAS Ring

Kirsten AMARAL

UTKARSH DOSHI

Albert P. LI

\section{Authorship Contributions}

Participated in research design: Ho, Doshi, Li.

Conducted experiments: Ho, Ring, Amaral, Doshi.

Performed data analysis: Ho, Ring, Doshi, Li.

Wrote or contributed to the writing of the manuscript: Ho, Ring, Amaral, Doshi, Li.

\section{References}

Bader A, Hansen T, Kirchner G, Allmeling C, Haverich A, and Borlak JT (2000) Primary porcine enterocyte and hepatocyte cultures to study drug oxidation reactions. $B r J$ Pharmacol 129: 331-342.

Bock KW (2016) The UDP-glycosyltransferase (UGT) superfamily expressed in humans, insects and plants: animal-plant arms-race and co-evolution. Biochem Pharmacol 99:11-17.

Bonnefille P, Sezgin-Bayindir Z, Belkhelfa H, Arellano C, Gandia P, Woodley J, and Houin G (2011) The use of isolated enterocytes to study Phase I intestinal drug metabolism: validation with rat and pig intestine. Fundam Clin Pharmacol 25:104-114.

Chen G, Zhang D, Jing N, Yin S, Falany CN, and Radominska-Pandya A (2003) Human gastrointestinal sulfotransferases: identification and distribution. Toxicol Appl Pharmacol 187: 186-197.

Chougule P, Herlenius G, Hernandez NM, Patil PB, Xu B, and Sumitran-Holgersson S (2012) Isolation and characterization of human primary enterocytes from small intestine using a novel method. Scand J Gastroenterol 47:1334-1343.

Crespi CL, Penman BW, and Hu M (1996) Development of Caco-2 cells expressing high levels of cDNA-derived cytochrome P4503A4. Pharm Res 13:1635-1641. 
Fabre G, Combalbert J, Berger Y, and Cano JP (1990) Human hepatocytes as a key in vitro model to improve preclinical drug development. Eur J Drug Metab Pharmacokinet 15:165-171.

Galetin A, Gertz M, and Houston JB (2008) Potential role of intestinal first-pass metabolism in the prediction of drug-drug interactions. Expert Opin Drug Metab Toxicol 4:909-922.

Gómez-Lechón MJ, Donato MT, Castell JV, and Jover R (2004) Human hepatocytes in primary culture: the choice to investigate drug metabolism in man. Curr Drug Metab 5:443-462.

Hansen T, Borlak J, and Bader A (2000) Cytochrome P450 enzyme activity and protein expression in primary porcine enterocyte and hepatocyte cultures. Xenobiotica 30:27-46.

Holmberg MT, Tornio A, Neuvonen M, Neuvonen PJ, Backman JT, and Niemi M (2014) Grapefruit juice inhibits the metabolic activation of clopidogrel. Clin Pharmacol Ther 95:307-313.

Jamei M, Turner D, Yang J, Neuhoff S, Polak S, Rostami-Hodjegan A, and Tucker G (2009) Population-based mechanistic prediction of oral drug absorption. AAPS J 11:225-237.

Kaminsky LS and Zhang QY (2003) The small intestine as a xenobiotic-metabolizing organ. Drug Metab Dispos 31:1520-1525.

Kolars JC, Schmiedlin-Ren P, Schuetz JD, Fang C, and Watkins PB (1992) Identification of rifampin-inducible P450IIIA4 (CYP3A4) in human small bowel enterocytes. J Clin Invest 90 1871-1878.

Küblbeck J, Hakkarainen JJ, Petsalo A, Vellonen KS, Tolonen A, Reponen P, Forsberg MM, and Honkakoski P (2016) Genetically modified Caco-2 cells with improved cytochrome P450 metabolic capacity. J Pharm Sci 105:941-949.

Lalovic B, Phillips B, Risler LL, Howald W, and Shen DD (2004) Quantitative contribution of CYP2D6 and CYP3A to oxycodone metabolism in human liver and intestinal microsomes. Drug Metab Dispos 32:447-454.

Li AP (2010) Evaluation of drug metabolism, drug-drug interactions, and in vitro hepatotoxicity with cryopreserved human hepatocytes. Methods Mol Biol 640:281-294.

Nakamura K, Hirayama-Kurogi M, Ito S, Kuno T, Yoneyama T, Obuchi W, Terasaki T, and Ohtsuki S (2016) Large-scale multiplex absolute protein quantification of drug-metabolizing enzymes and transporters in human intestine, liver, and kidney microsomes by SWATH-MS comparison with MRM/SRM and HR-MRM/PRM. Proteomics 16:2106-2117.

Nakamura T, Sakaeda T, Ohmoto N, Tamura T, Aoyama N, Shirakawa T, Kamigaki T, Nakamura T, Kim KI, Kim SR, et al. (2002) Real-time quantitative polymerase chain reaction for MDR1, MRP1, MRP2, and CYP3A-mRNA levels in Caco-2 cell lines, human duodenal enterocytes, normal colorectal tissues, and colorectal adenocarcinomas. Drug Metab Dispos 30:4-6.

Paine MF, Hart HL, Ludington SS, Haining RL, Rettie AE, and Zeldin DC (2006) The human intestinal cytochrome P450 "pie." Drug Metab Dispos 34:880-886.
Prueksaritanont T, Gorham LM, Hochman JH, Tran LO, and Vyas KP (1996) Comparative studies of drug-metabolizing enzymes in dog, monkey, and human small intestines, and in Caco-2 cells. Drug Metab Dispos 24:634-642.

Radominska-Pandya A, Little JM, Pandya JT, Tephly TR, King CD, Barone GW, and Raufman JP (1998) UDP-glucuronosyltransferases in human intestinal mucosa. Biochim Biophys Acta 1394 199-208.

Shirasaka Y, Shichiri M, Mori T, Nakanishi T, and Tamai I (2013) Major active components in grapefruit, orange, and apple juices responsible for OATP2B1-mediated drug interactions. $J$ Pharm Sci 102:280-288.

Teubner W, Meinl W, Florian S, Kretzschmar M, and Glatt H (2007) Identification and localization of soluble sulfotransferases in the human gastrointestinal tract. Biochem J 404:207-215.

Thummel KE (2007) Gut instincts: CYP3A4 and intestinal drug metabolism. $J$ Clin Invest 117: 3173-3176.

van de Kerkhof EG, de Graaf IA, Ungell AL, and Groothuis GM (2008) Induction of metabolism and transport in human intestine: validation of precision-cut slices as a tool to study induction of drug metabolism in human intestine in vitro. Drug Metab Dispos 36:604-613.

Vandel P, Haffen E, Vandel S, Bonin B, Nezelof S, Sechter D, Broly F, Bizouard P, and Dalery J (1999) Drug extrapyramidal side effects: CYP2D6 genotypes and phenotypes. Eur J Clin Pharmacol 55:659-665.

Wacher VJ, Salphati L, and Benet LZ (2001) Active secretion and enterocytic drug metabolism barriers to drug absorption. Adv Drug Deliv Rev 46:89-102.

Wang EJ, Casciano CN, Clement RP, and Johnson WW (2001) Inhibition of P-glycoprotein transport function by grapefruit juice psoralen. Pharm Res 18:432-438.

Watkins PB (1992) Drug metabolism by cytochromes P450 in the liver and small bowel. Gastroenterol Clin North Am 21:511-526.

Xie F, Ding X, and Zhang QY (2016) An update on the role of intestinal cytochrome P450 enzymes in drug disposition. Acta Pharm Sin B 6:374-383.

Zhang QY, Dunbar D, and Kaminsky LS (2003) Characterization of mouse small intestinal cytochrome P450 expression. Drug Metab Dispos 31:1346-1351.

Address correspondence to: Dr. Albert P. Li, 9221 Rumsey Road, Suite 8 Columbia, MD 21045. E-mail: lialbert1@invitroadmet.com 